\title{
Night blindness in primary biliary cirrhosis
}

\author{
Salman Waqar BSc MBBS, Thomas Kersey MBBS, Daniel Byles MB ChB
}

Previously published at www.cmaj.ca

A 58-year-old woman was referred by her optician because of decreased clarity of vision in both eyes with poor night vision. Her medical history included late-stage primary biliary cirrhosis. Her visual acuity was $6 / 6$ in both eyes and ocular examination was unremarkable. An electroretinogram showed a reduced amplitude scotopic (dark-adapted) response (Figure 1A) with a normal photopic (lightadapted) response. This finding suggested a vitamin A deficiency, and subsequent testing showed serum vitamin A levels were low at 0.3 (normal 0.7-1.7) $\mu \mathrm{mol} / \mathrm{L}$. She was given high-dose vitamin A supplementation orally with improvement in her symptoms and electroretinogram trace (Figure 1B).

Because of malabsorption of fat-soluble vitamins, vitamin A deficiency occurs in $33 \%$ of patients with primary biliary cirrhosis. ${ }^{1}$ Retinol is the most active form of vitamin $\mathrm{A}$ in the body. In the rod cells of the retina, all-trans retinol is converted into 11-cis retinol, which then combines with a membrane-bound protein called opsin to yield rhodopsin. Rhodopsin is essential for phototransduction, especially in low-light conditions. ${ }^{2}$ Thus patients with vitamin A deficiency (e.g., from malabsorption in primary biliary cirrhosis or extensive small bowel resection) may present with night blindness.

Electroretinography is primarily used in diagnosing retinal dystrophies and degeneration, evaluating chronic ischemic damage from vascular disease and monitoring drug toxicity (e.g., chloroquine, thioridazine). It is available in most ophthalmology departments. A corneal contact lens electrode is placed on the eye, which is well tolerated by most patients. The presence of cataracts does not appreciably affect testing.

The electroretinogram waveform represents a mass electrical response generated by the entire retina following a brief t flash of light. In general, the electroretinogram is characterized by a negative waveform (a wave) representing photoreceptor (rod and cone) response followed by a positive wave-

From the West of England Eye Unit, Royal Devon and Exeter NHS Foundation Trust, Exeter, United Kingdom

CMAJ 2010. DOI:10.1503/cmaj.090704 form (b wave) generated by the Müller cells and bipolar cell layer.

In photopic electroretinography, the patient stays in a light-adapted state and the retina is stimulated with a bright flash of light. The rods are thus suppressed by light adaptation and do not contribute to the waveform. The result is primarily a cone response with prominent $\mathrm{a}$ and $\mathrm{b}$ waves, and the results of this test were normal in our patient.

Because rods are 1000 times more sensitive to light than cones, dark-adapted testing or scotopic electroretinography is done by dark-adapting the patient's eyes for 20 minutes and then stimulating the retina with a dim light below the cone threshold. ${ }^{3}$ The resulting waveform normally has a prominent $\mathrm{b}$ wave and a minimal a wave. Because of rod dysfunction, vitamin A deficiency shows a flat scotopic electroretinogram, as was evident in our patient.

This article has been peer reviewed.

Competing interests: None declared.

Acknowledgement: The authors thank Mr. Paul Gerry for his help in obtaining electroretinograms.

\section{REFERENCES}

1. Phillips JR, Angulo P, Petterson T, et al. Fat-soluble vitamin levels in patients with primary biliary cirrhosis. Am J Gastroenterol 2001;96:2745-50.

2. Bok D. The retinal pigment epithelium: a versatile partner in vision. J Cell Sci Suppl 1993; 17:189-95.

3. American Academy of Ophthalmology. Basic and clinical science course 2008 2009 (retina and vitreous.) San Francisco (CA): American Academy of Ophthalmology; 2008. p. 33-5. 